\title{
THE NINETEENTH ANNUAL MEETING OF THE AMERICAN MATHEMATICAL SOCIETY.
}

The Society this year took advantage of the favorable opportunity afforded by the meeting of the American Association for the Advancement of Science at Cleveland to hold its annual meeting at that central point and to consolidate with it the usual winter meeting of the Chicago Section. Such occasions are always enjoyable and contribute greatly to solidify the common interests of the entire Society. Our members recall with pleasure the April meeting held at Chicago in 1911, and the long series of summer meetings and colloquia which bring together mathematicians from all parts of the country in united fellowship.

The time chosen for the annual meeting was Tuesday to Thursday, December 31 to January 2. Tuesday afternoon was set apart for a joint meeting with Sections A and B of the Association, the Astronomical and Astrophysical Society of America, and the American Physical Society. Separate sessions of the Mathematical Society were held on Tuesday morning, Wednesday morning and afternoon, and Thursday morning. The attendance included the following sixty-two members:

Professor O. P. Akers, Professor R. B. Allen, Professor Frederick Anderegg, Professor C. S. Atchison, Professor Clara L. Bacon, Professor W. W. Beman, Professor G. A. Bliss, Professor J. W. Bradshaw, Professor E. W. Brown, Professor W. H. Butts, Professor W. deW. Cairns, Professor F. N. Cole, Professor J. L. Coolidge, Professor D. R. Curtiss, Professor E. W. Davis, Professor L. E. Dickson, Professor H. T. Eddy, Professor J. A. Eiesland, Professor Arnold Emch, Professor G. C. Evans, Professor F. C. Ferry, Professor Peter Field, Professor J. C. Fields, Professor T. M. Focke, Professor W. B. Ford, Mr. Meyer Gaba, Professor W. A. Garrison, Mr. C. E. Githens, Professor M. E. Graber, Professor A. G. Hall, Professor Harris Hancock, Professor E. R. Hedrick, Dr. L. I Hewes, Professor L. S. Hulburt, Dr. W. A. Hurwitz, Professor A. M. Kenyon, Professor H. W. Kuhn, Dr. Joseph Lipke, Dr. Alexander Macfarlane, Dr. H. F. MacNeish, Professor 
W. H. Metzler, Professor G. A. Miller, Professor C. L. E. Moore, Professor C. N. Moore, Professor E. H. Moore, Professor Anna H. Palmié, Dr. H. B. Phillips, Professor S. E. Rasor, Professor H. L. Rietz, Professor W. J. Risley, Miss I. M. Schottenfels, Professor J. B. Shaw, Professor S. E. Slocum, Professor E. R. Smith, Professor K. D. Swartzel, Professor E. J. Townsend, Professor J. N. Van der Vries, Professor E. B. Van Vleck, Professor J. K. Whittemore, Professor E. J. Wilczynski, Dr. R. M. Winger, Professor J. W. Young.

The chair was occupied in succession by Professors E. W. Davis, E. H. Moore, G. A. Bliss, and after the annual election by the President-elect, Professor E. B. Van Vleck. The Council announced the election of the following persons to membership in the Society: Dr. E. W. Chittenden, University of Illinois; Mr. C. S. Cox, High School, Mulberry, Fla.; Dr. S. D. Killam, University of Rochester; Dr. J. T. Rorer, William Penn High School, Philadelphia, Pa.; Dr. R. M. Winger, University of Illinois. Sixteen applications for membership in the Society were received.

Sixty members and friends attended the annual dinner on Tuesday evening. A very informal dinner and smoker was also arranged for Wednesday evening. A vote of thanks was tendered to the local committee and the institutions represented by them for their generous hospitality.

The reports of the Treasurer, Auditing Committee, and Librarian have appeared in the Annual Register. The membership of the Society is now 681, including 64 life members. The total attendance of members at all meetings of the past year was 336; the number of papers presented was 179 . At the annual election 216 votes were cast. The Society's library now contains 4,560 volumes, excluding unbound dissertations. Much of this considerable increase is due to gifts by Dr. Emory McClintock and Dr. G. W. Hill, Ex-Presidents of the Society, of several hundred valuable volumes. The Treasurer's report shows a balance of $\$ 9684.92$, including a life membership fund of $\$ 4483.69$. The income from sales of the Society's publications during the year was $\$ 1730.94$.

At the annual election, which closed on Thursday morning, the following officers and other members of the Council were chosen: 
President, $\quad$ Professor E. B. VAN VLECK.

Vice-Presidents, Professor M. W. Haskell, Professor B. O. Peirce.

Secretary, Professor F. N. Cole.

Treasurer, Professor J. H. TANner.

Librarian, $\quad$ Professor D. E. SMITH.

Committee of Publication,

Professor F. N. Cole,

Professor E. W. Brown,

Professor VIRGIL SNYDER.

Members of the Council to serve until December, 1915,

Professor F. C. Ferry, President R. C. Maclaurin, Professor W. B. Ford, Professor JACOB Westlund.

The following papers were read at the joint session on Tuesday:

Professor E. B. Frost; Vice-presidential address, Section A: "The spectroscopic determination of stellar velocities, considered practically.",

Professor R. A. Millikan; Vice-presidential address, Section B: Unitary theories in physics."

Professor A. G. Webster: "Henri Poincaré as a mathematical physicist."

Professor E. J. Wilczynskr: "Some general aspects of modern geometry."

Professor L. A. BAuer: "Cosmical magnetic fields."

Professor G. E. HALE: "Preliminary note on an attempt to detect the general magnetic field of the sun."

The following papers were read at the separate sessions of the Society:

(1) Professor R. D. Carmichael: "On the numerical factors of the arithmetic forms $\alpha^{n} \pm \beta^{n}$."

(2) Professor R. D. Carmichael: "On non-homogeneous linear equations with an infinite number of variables."

(3) Professor R. D. Carmichael: "Note on Fermat's last theorem."

(4) Dr. W. A. Hurwitz: "Mixed linear integral equations of the first order." 
(5) Mr. H. Galajikian: "On certain non-linear integral equations."

(6) Dr. W. A. Hurwitz: "On Green's theorem for the plane."

(7) Professor ARNold EMch: "On some properties of closed continuous curves."

(8) Professor G. A. Miller: "The product of two or more groups."

(9) Dr. J. E. Rowe: "Three or more rational curves in collinear relation."

(10) Professor F. R. Sharpe and Dr. F. M. Morgan: "Quartic surfaces invariant under periodic transformations."

(11) Dr. H. M. Sheffer: "A set of postulates for the Boolean algebra."

(12) Dr. J. R. Conner: "The rational sextic curve and the Cayley symmetroid."

(13) Dr. J. R. Conner: "Multiple correspondences determined by the rational space septimic."

(14) Professor L. E. Dickson: "Finiteness of the odd perfect and primitive abundant numbers with a given number of distinct prime factors."

(15) Professor L. E. Dickson: "Amicable number triples."

(16) Professor J. L. Coolidge: "A study of the circlecross."

(17) Professor G. A. BuIss: "The relation satisfied by two dependent functions near a point at which both are singular."

(18) Professor J. A. Eiesland: "On the algebraic curves of a tetrahedral complex and the corresponding surfaces conjugate to it."

(19) Professor E. H. Moore: "On nowhere negative kernels" (preliminary communication).

(20) Professor Daniel Buchanan: "Oscillations near one of the isosceles triangular solutions of the three body problem."

(21) Professor Peter Field: "On constrained motion."

(22) Professor G. C. Evans: "On the reduction of certain types of integro-differential equations."

(23) Professor J. A. Caparo: "Hyperspace and the noneuclidean geometry of four dimensions."

(24) Professor JACOB Westuund: "On the factorization of rational primes in cubic cyclotomic number fields."

(25) Dr. E. L. Dodd: “An erroneous application of Bayes' theorem to the set of real numbers." 
(26) Dr. E. L. DodD: "The validity of Bertrand's approximations leading to the probability integral."

(27) Professor EDwaRD KASNER: "Equitangential trajectories in space."

(28) Professor C. J. Keyser: "Concerning multiple interpretations of postulate systems, and the 'existence' of hyperspaces."

(29) Professor E. J. Wilczynski: "On a certain completely integrable system of linear partial differential equations."

(30) Professor L. C. KarPinski: "The Quadripartitum numerorum of Johannes de Muris."

(31) Professor L. C. KarPinski: "Hindu numerals among the Arabs."

(32) Dr. H. B. Phillips: "Directed integration."

(33) Dr. Joseph LIPKE: "Geometric characterization of isogonal trajectories on a surface."

(34) Professor J. B. SHAw: "Integral invariants of general vector analysis."

(35) Professor J. B. SHAw: "On non-linear algebras."

(36) Professor D. R. CuRtiss: "Proofs of certain formulas suggested by Laguerre's work in the theory of equations."

(37) Professor Arthur Ranum: "On the projective differential classification of $n$-dimensional spreads generated by $\infty^{1}$ flats."

(38) Miss I. M. Schottenfels: "Proof that there is but one simple group of order $7 ! / 2 . "$

(39) Professor L. P. Eisenhart: "Certain continuous deformations of surfaces applicable to the quadrics."

(40) Professor E. V. Huntington: "A set of independent postulates for 'betweenness.",

(41) Professor A. B. Frizell: "Some terms in the expansion of the infinite determinant."

(42) Dr. T. H. Gronwall: "On Weierstrass's preparation theorem."

(43) Dr. T. H. Gronwall: "On series of spherical harmonics (second paper)."

(44) Dr. CorA B. Hennel: "Transformations and invariants connected with linear homogeneous difference equations and other functional equations."

(45) Professor Harris Hancock: "Problems in arithmetical geometry."

(46) Professor Harris Hancock: "Generalization of a 
theorem due to Liouville or to Dedekind, with applications to the geometry of numbers."

(47) Professor W. D. MacMillan: “A proof of Wilczynski's theorem."

(48) Professor W. D. MacMillan: "On Poincarés correction to Bruns' theorem."

(49) Professor W. B. Fite: "Some theorems concerning groups whose orders are powers of a prime."

(50) Mr. L. L. Smail: "Some generalizations in the theory of summable divergent series."

(51) Mr. C. E. Love: "On the asymptotic solutions of linear differential equations."

(52) Professor Virgil Snyder: "Algebraic surfaces invariant under an infinite discontinuous group of birational transformations (second paper)."

(53) Dr. L. L. Silverman: "On the equivalence of definitions of summability."

(54) Dr. R. M. Winger: "Self-projective rational curves of the fourth and fifth orders."

The papers of Mr. Galajikian and Dr. Sheffer were communicated to the Society through Dr. Hurwitz, that of Professor Caparo through Professor G. A. Miller. Dr. Hennel was introduced by Professor Carmichael, Mr. Love by Professor Ford. The papers of the following authors were read by title: Professor Carmichael, Professor Miller, Dr. Rowe, Professor Sharpe and Dr. Morgan, Dr. Sheffer, Dr. Conner, Professor Buchanan, Professor Evans, Professor Caparo, Professor Westlund, Dr. Dodd, Professor Kasner, Professor Keyser, Professor Ranum, Professor Eisenhart, Professor Huntington, Professor Frizell, Dr. Gronwall, Professor MacMillan, Professor Fite, Mr. Smail, Professor Snyder, Dr. Silverman. The second papers of Professor Dickson and Professor Karpinski were also read by title. Mr. Galajikian's paper was read by Dr. Hurwitz.

Abstracts of the papers follow below. The abstracts are numbered to correspond to the titles in the list above.

1. Let $\alpha$ and $\beta$ be the roots of the quadratic equation

$$
z^{2}-(\alpha+\beta) z+\alpha \beta=0,
$$

whose coefficients $\alpha+\beta$ and $\alpha \beta$ are relatively prime integers such that $\alpha$ and $\beta$ are not roots of unity. Then the numbers 
$D_{n}$ and $S_{n}$,

$$
D_{n}=\frac{\alpha^{n}-\beta^{n}}{\alpha-\beta}, \quad S_{n}=\alpha^{n}+\beta^{n}
$$

are integers. The principal object of Professor Carmichael's first paper is an investigation of the numerical properties of $D_{n}$ and $S_{n}$. The general results obtained have several applications in the theory of numbers.

Among the factors of $D_{n}$ and $S_{n}$ are numbers of the form $F_{k}(\alpha, \beta)$, where $F_{k}(\alpha, \beta)$ is that irreducible algebraic factor of $\alpha^{k}-\beta^{k}$ which is not a factor of any $\alpha^{\nu}-\beta^{\nu}$ for which $\nu$ is less than $k$. These numbers $F_{k}(\alpha, \beta)$ play a fundamental rôle throughout the paper.

The general results cannot be briefly stated. Of the conclusions obtained in application of the general theorems we mention the following two: a necessary and sufficient condition that an odd number $p$ is prime is that an integer $a$ exists such that $F_{p-1}(a, 1)$ is divisible by $p$; a necessary and sufficient condition that $2 k+1=2^{2^{n}}+1$, where $n>1$, is prime is that

$$
3^{k}+1 \equiv 0 \bmod 2 k+1
$$

2. In recent years important contributions to the problem of solving linear equations with an infinite number of variables have been made by Hill, Poincaré, von Koch, Hilbert, Toeplitz, Schmidt, and Bôcher and Brand. The most interesting and far-reaching developments which have been given up to the present time are those of the last two mentioned. 'The object of Professor Carmichael's second paper is to establish a range of validity for results obtained by a useful method due to Kötteritzsch.

The method of the paper, although it is one of great simplicity, is yet such as not to yield readily to description within limits of space appropriate for this abstract.

3. Professor Carmichael's third paper appeared in full in the February Bulletin.

4. In his first paper Dr. Hurwitz attempts to supply a relatively complete theory of mixed linear equations of the first order, such as he has considered briefly in an earlier note. 
The notion of the adjoint system to such an equation is introduced and used to obtain a simple form of statement of conditions for the solution of the non-homogeneous equation in cases where the homogeneous equation possesses nontrivial solutions. A function $D(\lambda)$ corresponds to the Fredholm determinant of the pure equation, and a set of functions $D(x, y ; \lambda) ; D_{1}(x ; \lambda), D_{2}(x ; \lambda), \cdots, D_{m}(x ; \lambda)$ corresponds to the Fredholm first minor; these are integral functions of $\lambda$. Resolvent and pseudo-resolvent systems may be constructed, having properties analogous to those of the resolvent and pseudo-resolvent functions for the pure equation. By means of a natural generalization a parallel to the theory of the symmetric kernel may also be established.

5. In this paper Mr. Galajikian considers integral equations of the type

$$
y(x)=g\left\{x, \int_{x_{0}}^{x} f_{1}(x, t, y(t)) d t, \cdots, \int_{x_{0}}^{x} f_{m}(x, t, y(t)) d t\right\},
$$

with the generalization to systems of such equations. By the method of successive approximations it is shown that a continuous solution exists in a sufficiently small interval under conditions of continuity and Lipschitz conditions on the given functions. A special form of the equation considered has been treated by $\mathrm{T}$. Lalesco.

6. The usual proofs of Green's theorem on the equality of certain double and curve integrals not only demand that the region of double integration be bounded by simple regular closed curves, but also explicitly or implicitly use a further assumption, ordinarily in the form that the boundary should be cut in only a finite number of points by any straight line parallel to either coördinate axis. As results are frequently stated, in the application of the theorem to partial differential equations, without mention of this restriction, one is led to ask whether or not it is essential. Dr. Hurwitz in his second paper establishes the truth of Green's theorem without the additional assumption on the boundary.

7. In a paper recently presented to the Southwestern Section Professor Emch has shown how to represent para- 
metrically any closed continuous curve passing through the vertices of a square. He has proved also that at least one square may be inscribed in every ordinary (admitting of a definite tangent at every point) continuous closed convex curve or oval. From this follows that any oval may be parametrically represented in the form referred to above.

In the present paper the results are generalized for any ordinary closed curve without singular points.

8. Professor Miller's paper appears in full in the present number of the BULLETIN.

9. Certain rational plane curves possess sets of covariant rational point or line curves which are related as follows: if $A, B$, and $C$ are three point curves of such a set, any parameter value substituted in the parametric equations of $A, B$, and $C$ yields the coördinates of three collinear points; if $A^{\prime}$, $B^{\prime}$, and $C^{\prime}$ are three line curves of such a set, any parameter value substituted in the parametric equations of $A, B$, and $C$ yields the coördinates of three concurrent lines; Dr. Rowe's paper consists in discussing such curves, and in giving illustrations of their existence.

10. Professor Sharpe and Dr. Morgan consider a quartic surface having two conical points $P$ and $Q$. If any line through $P$ meets the surface in $A$ and $B$ and $Q B$ meets the surface in $C$, the conditions are found that the transformation which sends $A$ into $C$ should be of period 2 or 3 . There appears to be no surface of this type with a transformation of period $>3$ for all positions of $A$. The conditions may be interpreted as the condition that the two involutorial transformations $I_{1}, I_{2}$ of the general $(2,2)$ correspondence should satisfy $\left(I_{1} I_{2}\right)^{2}$ or $\left(I_{1} I_{2}\right)^{3} \equiv 1$. The geometrical interpretation for a quartic curve with two double points is given and is analogous to Steiner's theorems for a cubic curve (Crelle, 1845).

11. In this paper Dr. Sheffer gives a determination of the Boolean algebra, the "algebra of logic," by means of a set of postulates which differs from Huntington's sets (Transactions, volume 5, pages 288-309) mainly in that Huntington's existence postulates for the logical elements 0 and 1 , and for the class of "negatives," are deduced as theorems. 
12. This paper is intended primarily as an illustration of the value of the rational curve as an instrument by means of which the properties of certain types of surfaces may be studied. Dr. Conner's starting-point is the rational sextic curve $r^{6}$,

$$
\xi_{i}=\left(\alpha_{i} t\right)^{6} \quad(i=1,2,3,4),
$$

taken in connection with its conjugate $\rho^{6}$,

$$
x_{i}=\left(a_{i} t\right)^{6} \quad(i=1,2,3,4),
$$

where $\left|\alpha_{i} a_{j}\right|^{6}=0$. Giving a point $x$ of space determines 6 parameters on $r^{6}$; if the catalecticant of the binary sextic so obtained is made to vanish, the locus of $x$ is the quartic surface $S$ with 10 symmetrical nodes, called by Cayley the symmetroid. The rational curve $r^{6}$ and the symmetroid have each 24 constants, and it is shown that $S$ has two associated rational sextics, each of which determines it in the above manner. These sextics have a common rational covariant quadric surface $K$, which is thus a rational covariant surface of the symmetroid. The separation of the sextics depends on the separation of the two systems of generators of $K$.

There are 10 quadrics each on all nodes of $S$ but one, and the two systems of generators of each of these pair off with the two systems on $K$. Thus giving one of these latter quadrics a node forces every other to have a node-a remarkable property of the 10 nodes of $S$.

Other covariant surfaces and special cases of $S$ (Kummer surface, Hessian of cubic) are discussed.

13. This paper is to be regarded as a continuation of an earlier paper by Dr. Conner, entitled "Multiple correspondences determined by the rational plane quintic curve" (Transactions, volume 13, pages 265-275, April, 1912).

Given a rational 7 -ic curve $\rho^{7}$ in a space $S$,

$$
x_{i}=\left(a_{i} t\right)^{7} \quad(i=1,2,3,4)
$$

and its conjugate curve $r^{7}$ in a space $\Sigma$,

$$
\xi_{i}=\left(\alpha_{i} t\right)^{7} \quad(i=1,2,3,4),
$$

where $\left|a_{a} \alpha_{j}\right|^{7}=0$, a $(7,1)$ correspondence $T$ is determined between $S$ and $\Sigma$. The surfaces in $S$ which are maps by $T$ of 
planes of $\Sigma$ are a 3 -fold linear system of cubic surfaces $C_{3}$, having a common double line $\pi$. The points of $\pi$ are the only singular points of $\Sigma$. The jacobian $J$ of the system $C_{3}$ is transformed by $T$ into the developable of tangents of $r^{7}$. $T$ transforms planes of $S$ into quintic surfaces in $\Sigma$ which bear interesting relations to the surface $T(J)$. Other correspondences associated with $T$ are discussed.

The proof of the existence of $T$ and its connection with the osculants of $\rho^{7}$ are developed in a manner analogous to the method of the earlier paper.

It is shown that similar correspondences exist for rational curves in general, and it is hoped that their value as an aid to the study of rational curves is clearly shown.

14. In the first paper by Professor Dickson a primitive non-deficient number is defined to be a non-deficient number not a multiple of a smaller non-deficient number. It is proved that there is only a finite number of primitive nondeficient odd numbers having any given number $n$ of distinct prime factors. Since any perfect number is a primitive non-deficient number, it follows as a corollary that there is not an infinitude of odd perfect numbers with a given number of distinct prime factors. The main theorem is proved by applying the following lemma once to the set of all deficient numbers with given prime factors (to prove by induction that the $n$ prime factors of a primitive non-deficient number are each limited) and finally to the set of all primitive non-deficient numbers with $n$ given prime factors. The lemma states that any set $S$ of integers $p_{1}^{e_{1}} \cdots p_{n}{ }_{e_{n}}$, where $p_{1}, \cdots, p_{n}$ are given integers, contains a finite number of integers $F_{1}, \cdots, F_{k}$ such that every integer of the set is a multiple of at least one $F_{i}$. By the same method it is shown that there is only a finite number of primitive abundant numbers having a given number of distinct odd prime factors and a given number of factors 2 . The paper will be offered to the American Journal of Mathematics.

15. In the second paper by Professor Dickson an amicable number triple is defined to be a set of three integers such that the sum of the proper divisors of each equals the sum of the remaining two numbers. If $\sigma(n)$ is the sum of all the divisors of $n$, then $n_{1}, n_{2}, \cdots, n_{k}$ form an amicable $k$-tuple if

$$
\sigma\left(n_{1}\right)=\sigma\left(n_{2}\right)=\cdots=\sigma\left(n_{k}\right)=n_{1}+n_{2}+\cdots+n_{k} .
$$


For $k=2$, the numbers are amicable in the usual sense. If the $n$ 's are all equal, $n_{1}$ is a multiply perfect number of multiplicity $k$. For $k=3$, the paper gives 7 amicable triples in which two of the three numbers are equal, and the two examples of amicable triples of distinct numbers

$$
\begin{aligned}
& 293.337 a, \quad 5 \cdot 16561 a, \quad 99371 a, \quad a \equiv 2^{5} \cdot 3 \cdot 13 ; \\
& 3 \cdot 89 a, \quad 11 \cdot 29 a, \quad 359 a, \quad a \equiv 2^{14} \cdot 5 \cdot 19 \cdot 31 \cdot 151 .
\end{aligned}
$$

The paper will appear in the American Mathematical Monthly.

16. A circle-cross is the figure of two circles so related that each is orthogonal to every sphere through the other. It bears a relation to the circles of a general linear system analogous to that which the central axis bears to the lines of a linear complex. Professor Coolidge's paper considers systems of crosses orthogonal to one sphere, crosses derived from one and two parameter families of linear circle systems, and an involutory transformation which carries circles into circles, but not spheres into spheres.

17. It is well known that when the functional determinant of two analytic functions $\varphi(u, v)$ and $\psi(u, v)$ vanishes identically there must be a relation of the form

$$
F(\varphi(u, v), \psi(u, v)) \equiv 0,
$$

holding identically in $u$ and $v$. Near any point which is not a singular point for $\varphi$, the form of this relation can be found by solving the equation

$$
\varphi(u, v)=k
$$

for one of the variables and substituting the result in $\psi$. Near a point at which both $\varphi$ and $\psi$ are singular this process can not be carried out. In the paper of Professor Bliss a method is given for determining the character of the relation near a point where $\varphi$ and $\psi$ both begin with terms of higher than the first degree, and it is shown that $F(\varphi, \psi)$ is analytic but has itself a singular point. The proof makes use of the preparation theorem of Weierstrass by means of which the variable $u$ can be eliminated from the functions $\varphi-x, \psi-y$. The resultant $R(v, x, y)$ is a series in $v, x, y$, and the function $F(\varphi, \psi)$ required is $R(0, \varphi, \psi)$. 
18. The purpose of Professor Eiesland's paper is to determine all the algebraic curves of a tetrahedral complex. The algebraic surfaces conjugate to the complex may then, according to Lie, be formed by putting

$$
\begin{aligned}
& \log x=\int \frac{U d u}{a+u}+\int \frac{V d v}{a+v}, \\
& \log y=\int \frac{U d u}{b+u}+\int \frac{V d v}{b+v}, \\
& \log z=\int \frac{U d u}{c+u}+\int \frac{V d v}{c+v},
\end{aligned}
$$

where $(u)$ and $(v)$ are complex curves and also conjugate curves on the surface. The work of determining these curves (and surfaces) is chiefly of a function-theoretical nature, but a number of interesting geometrical theorems have also been found in the course of the investigation.

19. In his preliminary communication Professor Moore stated the following theorems:

(1) If a real-valued continuous kernel $\kappa(s, t)(a \leqq s \leqq b$, $a \leqq t \leqq b$ ) satisfies the two conditions: ( $a$ ) for every realvalued continuous function $\xi(s)$ not identically null $(a \leqq s \leqq b)$

$$
\int_{a}^{b} \xi(s) \xi(s) d s>\int_{a}^{b} \int_{a}^{b} \xi(s) \kappa(s, t) \xi(t) d s d t ;
$$

(b) $\kappa(s, t)$ is nowhere negative, then the kernel $\kappa(s, t)$ has no characteristic number of absolute value $\leqq 1$ - Corollaries:

(2) For such a kernel $\kappa(s, t)$ the solution $\eta(s)$ of the linear integral equation

has the form

$$
\xi(s)=\eta(s)-\int_{a}^{b} \kappa(s, t) \eta(t) d t \quad(a \leqq s \leqq b),
$$

$$
\begin{aligned}
\eta(s)=\xi(s) & +\int_{a}^{b} \kappa(s, t) \xi(t) d t \\
& +\int_{a}^{b} \int_{a}^{b} \kappa(s, t) \kappa(t, u) \xi(u) d t d u+\cdots \quad(a \leqq s \leqq b),
\end{aligned}
$$

and hence 
(3) If the given function $\xi(s)$ is nowhere negative or everywhere positive $(a \leqq s \leqq b)$, the solution $\eta(s)$ is respectively nowhere negative or everywhere positive $(a \leqq s \leqq b)$.

According to Hilbert and Bateman, the conclusion of (1) holds for symmetric kernels with the condition $(a)$. In this case the characteristic numbers are real. For (possibly asymmetric) kernels with the conditions $(a, b)$ one proves readily that a real characteristic number, if any exists, is of absolute value $>1$, and hence that every characteristic number is of absolute value $>1$, from the theorem:

(4) For a kernel $\kappa(s, t)$ with the condition (b) either no characteristic number exists or there exists a positive characteristic number amongst the characteristic numbers of least absolute value. This follows from Fredholm's expression of the logarithm of the integral transcendental function $D(\lambda)$ as a power series in $\lambda$ convergent near $\lambda=0$.

The theorems here stated are instances of general theorems in Professor Moore's general theory of linear integral equations (cf. this Bulletrin, April 1912). Theorem (3) for the symmetric (algebraic) instance $\mathrm{II}_{n}$ of a system of $n$ linear equations in $n$ unknowns $\eta(s) \quad(s=1,2, \cdots, n)$ is essentially a theorem established by Stieltjes (Acta Mathematica, volume 9, page 385,1887$)$ for use in his investigation of the roots of Legendre's polynomials.

20. The isosceles triangular solutions of the three body problem are the periodic solutions in which two of the masses are finite and equal, while the third body moves so that it is equidistant from the finite bodies. Pavanini obtained the first of these solutions in 1907 by means of an elliptic integral. In this solution the finite bodies move in a circle and the third body, an infinitesimal, moves in a line through the center of mass perpendicularly to the plane of the motion of the finite bodies. Macmillan also obtained this solution independently in 1910, and further developed the solution as a periodic function of the independent variable. In his paper before the society at the April meeting in Chicago, 1911, Professor Buchanan dealt with two additional solutions: one in which the finite bodies move in ellipses and the third body is infinitesimal; the other in which the three bodies are finite. The present paper deals with the case in which the infinitesimal body oscillates about the straight line while the equal bodies 
move in a circle. The solution is developed as a power series in two parameters, one representing the initial projection from the plane of motion of the finite bodies, the other representing the initial displacement from the center of mass.

21. The suggestion of studying the type of constrained motion which is considered in Professor Field's paper was obtained from an article by Saint-Germain and Lecornu entitled "Sur l'impossibilité de certaines mouvements," published in the Comptes Rendus, volume 114 (1892).

The following problem is solved. Given two parallel plane curves $c$ and $c_{1}$, the distance between the two being $a$; two particles of mass $m$ and $m_{1}$, connected by a weightless rod of length $a$, are constrained to move along $c$ and $c_{1}$. No external forces act on the particles. Under what circumstances does the supposition that motion takes place lead to conditions which are compatible: $(a)$ when $c$ and $c_{1}$ are smooth, $(b)$ when $c$ and $c_{1}$ are rough?

22. We are often concerned, as in the theory of Galois, with the question as to when the solution of certain equations can be expressed in terms of a finite number of operations and functions, belonging to given classes. We may speak of this as the question of getting the solutions in closed form.

Professor Evans considers the closed forms of solutions of certain types of linear integro-differential equations. By means of transformations involving a finite number of quadratures, the integro-differential equations in these cases are reduced to systems of equations purely differential or purely integral.

23. From considerations of the non-euclidean geometries of Riemann and Lobachevsky it can be demonstrated that the space of our physical perceptions could have a curvature, although very small, towards a fourth dimensional space, and though this cannot be proved by experiment upon space, still its consideration involves a philosophical train of thought and a series of useful principles in the realm of pure mathematics. In Professor Caparo's paper several hyperbodies are studied under the most simple conditions; e. g., hyperbodies extending from a three-dimensional space towards the space of four dimensions, thus leaving their bases, or at least their sections, in the three dimensional space. 
Relations between spaces of higher order and the orders of infinite are also established, showing the non-absurdity of the assumptions and conclusions in the proposed theorems and axioms of a non-euclidean fourth dimensional geometry. The degrees of freedom of elements in hyperspaces are also studied with a view of explaining several physical and chemical phenomena.

24. If $p$ is an odd prime of the form $6 n+1, r$ a primitive $p$ th root of unity, and $g$ a primitive root of $p$, then $\alpha=r$ $+r^{g 3}+\cdots+r^{g}{ }^{(2 n-1) 3}$ generates a cubic cyclic number field. In order to determine the class number of this field a knowledge of the prime ideals in the field is necessary. In Professor Westlund's paper a method for decomposing rational primes into prime ideal factors in $k(\alpha)$ is given, and the method is applied, for a large number of values of $p$, to the actual factorization of those rational primes on which the determination of the class number depends.

25. Dr. Dodd deals with an attempted generalization of the following corollary of Bayes' theorem, viz.: If each of a finite number of mutually exclusive "causes" is equally likely, a priori, to come into play, then the probability, a posteriori, that a given event had its origin in a given cause is proportional to the probability that the given cause would produce that event.

In the theory of measurements a function $\psi(z) d z$ is sometimes regarded as giving the probability, a priori, that the true value lies between $z$ and $z+d z$. Then the attempt is made to regard $\psi(z)$ as a constant for all real values of $z$, viewing each real number as equally likely, a priori, to be the true value. Not only is this probability difficult to interpret in terms of ideal frequency, but $\psi(z)$ cannot be made a constant; for the integral of $\psi(z)$ from $-\infty$ to $+\infty$ must be unity, the symbol for certainty.

With measurements designated by $m_{1}, m_{2}, \cdots, m_{n}$, true value by $z$, and errors by $z-m_{1}, \cdots, z-m_{n}$, the probability that this specified set of measurements, with attendant errors, will occur is written as

$$
P=\varphi\left(z-m_{1}\right) \varphi\left(z-m_{2}\right) \cdots \varphi\left(z-m_{n}\right),
$$


or perhaps this product multiplied by $d m_{1} \cdots d m_{n}$. The attempt is then made to view $P$ as a function of $z$, proportional to the probability that $z$ be the true value,-it being imagined that this is a legitimate generalization of the corollary of Bayes' theorem-when, indeed, the writer seeks any justification at all. After hasty reading, some may even suppose that $P(z)$ is equal to the probability that $z$ be the true value.

Then setting $d P / d z=0$, and assuming that the arithmetic mean is the most probable value, the argument is supposed to lead to the Gaussian probability law.

26. Dr. Dodd uses Stirling's formula in finite form, ${ }^{*}$ viz.: $n !=n^{n} e^{-n+\frac{\theta}{12 n}} \sqrt{2 \pi n}$, where $0<\theta<1$, and finite developments of $e^{t}$ and $\log _{e}(1+t)$, to verify Bertrand's $\dagger$ deduction of the probability integral.

Let $p$ be the probability that an event will happen on a single trial, where $0<p<1$; and set $q=1-p$. Then the probability that the event will happen exactly $r$ times in $s$ trials is

$$
P_{r}=\frac{s !}{r !(s-r) !} p^{r} q^{s-r} .
$$

Now let any positive number $c$ be chosen; and then let $s$ be taken large enough so that $s p-c \sqrt{s}>0$ and $s p+c \sqrt{s}$ $<s$. Then let $r, r_{1}$, and $r_{2}$ be such that

$$
s p-c \sqrt{s} \leqq r_{2} \leqq r \leqq r_{1} \leqq s p+c \sqrt{s} .
$$

Set

$$
l=s p-r, \quad l_{1}=s p-r_{1}, \quad l_{2}=s p-r_{2}, \quad h=(2 s p q)^{-\frac{1}{2}} .
$$

Then constants, $K_{1}, K_{2}, K_{3}$, and $K_{4}$, independent of $s$, exist such that

$$
\begin{gathered}
P_{r}=\frac{h}{\sqrt{\pi}} e^{-h^{2} l^{2}}+\epsilon_{1}, \quad\left|\epsilon_{1}\right|<\frac{K_{1}}{s} ; \\
\sum_{r_{2}}^{r_{1}} P_{r}=\frac{h}{\sqrt{\pi}} \sum_{l_{1}}^{l_{2}} e^{-h^{2} l^{2}}+\epsilon_{2}, \quad\left|\epsilon_{2}\right|<\frac{K_{2}}{s} ;
\end{gathered}
$$

* Cesàro, Corso di Analisi algebrica, pp. 270 and 480.

† Calcul des Probabi lités, p. 76. 


$$
\begin{aligned}
\frac{h}{V^{\prime} \pi} e^{-h^{2} l^{2}} & =\frac{h}{\sqrt{\pi}} \int_{l-1 / 2}^{l+1 / 2} e^{-h^{2} x^{2}} d x+\epsilon_{3}, & \left|\epsilon_{3}\right|<\frac{K_{3}}{s \sqrt{s}} ; \\
\sum_{r_{2}}^{r_{1}} P_{r} & =\frac{h}{\sqrt{ } \pi} \int_{l-1 / 2}^{l+1 / 2} e^{-h^{2} x^{2}} d x+\epsilon_{4}, & \left|\epsilon_{4}\right|<\frac{K_{4}}{\sqrt{s}}
\end{aligned}
$$

It can now be shown that $\lim _{s=\infty} \epsilon_{4}=0$, uniformly, even with the restriction (2) removed.

Theorem.-Let $p$ be the probability that an event will occur on a single trial, where $0<p<1$; let $s$ be the number of trials to be made; and set $h=[2 s p(1-p)]^{-\frac{1}{2}}$. Then the probability that the number of occurrences of the event will be some number in the set $s p-l_{2}$ to $s p-l_{1}$ inclusive, where $0 \leqq s p-l_{2} \leqq s p-l_{1} \leqq s$, is

$$
\frac{1}{\sqrt{ }}=\int_{h l_{1}}^{h l_{2}} e^{-t^{2}} d t+\delta, \quad \text { where } \lim _{s=\infty} \delta=0 \text {, uniformly. }
$$

27. If each lineal element of a congruence of space curves slides along its own direction a fixed distance $c$, the new elements generate a new congruence equitangentially related to the original. By varying $c$, we obtain a family of $\infty^{3}$ space curves, whose properties are discussed by Professor Kasner. The simplest results are the following. A line tangent to one curve is tangent to $\infty^{1}$ curves; the osculating planes passing through the line are homographically related to the points of contact; the locus of the centers of curvature is a twisted cubic.

28. Professor Keyser's paper, which for definiteness attaches itself to Hilbert's axioms for geometry, undertakes to examine the current "critical" creed: that the element-names, since they are not defined, may be interpreted to be the names of any things whatever, subject to the sole restriction that the things must satisfy the axioms; that, an admissible (possible) interpretation once given, a definite science, theory, or doctrine arises; that replacing that interpretation by another admissible one leaves the doctrine unchanged; that this doctrine is euclidean geometry of three dimensions; and that, if one wishes to regard the geometry as a doctrine of or about something to be called space $S_{3}, S_{3}$ is nothing but any system of 
things that satisfy the axioms. The examination leads to the conclusion that, whilst there are many (even infinitely many) admissible interpretations, no two of these yield the same doctrine; that one and but one of the doctrines so arising is rightly called geometry; that it is not merely a matter of temperament or taste whether this doctrine be regarded as being about something (called space); that this space, though it is not sensible (or empirical) space, is an affair connoting conceptual extension, as Euclid's much ill-advisedly criticised descriptive "definitions" of point, line and plane so usefully intimate; and that, even if this extensional view of space be rejected, the long vexed question as to what, if any, sort of existence hyperspaces have, is to be answered finally by the proposition that hyperspaces possess every kind of existence that may be warrantably attributed to the space of ordinary geometry.

29. In a paper read before the summer meeting of the Society, Professor Wilczynski defined a class of surfaces whose theory was equivalent to the theory of the completely integrable system

$$
\begin{aligned}
& \frac{\partial^{2} y}{\partial u^{2}}+2 \frac{\partial y}{\partial v}+\left(c_{0} u+c_{1}\right) y=0 \\
& \frac{\partial^{2} y}{\partial v^{2}}+2 \frac{\partial y}{\partial u}+\left(c_{0} v+c_{2}\right) y=0
\end{aligned}
$$

where $c_{0}, c_{1}$, and $c_{2}$ are constants. An exhaustive discussion was there given of the cases in which $c_{0}$ is equal to zero. The present paper is devoted to the integration of the above system in the case where $c_{0}$ does not vanish. One of the preliminaries to the solution of this problem is the problem of integrating a completely integrable non-homogeneous system when the integrals of the corresponding homogeneous system are known. The integrals of $(S)$, which then appear as power series in $c_{0}$, have associated with them a remarkable family of polynomials whose properties are studied to some extent. It turns out, moreover, that the solutions of $(S)$ satisfy integral equations of a peculiar kind, involving exact differentials in two independent variables, thus suggesting several new problems to which, in part at least, the processes of this paper are applicable. 
30. This is a final report on the Quadripartitum, more especially of the third book dealing with algebra. Professor Karpinski shows that de Muris drew extensively both from Leonard of Pisa and from Al-Khowarizmi. While this writer contributed little if anything to the development of the science, yet his work was influential in spreading the popular study of algebra. The origin of certain terms used for algebra, ars major and ars rei et census, is touched.

31. Up to the present time the earliest known forms of Hindu numerals among the Arabs are in documents of 873 A.D. The arithmetic of Al-Khowarizmi antedates this by some fifty years, but it has not yet been found in the Arabic original but only in Latin translation. Professor Karpinski presents a reference to the numerals in an Arabic work of 855 A.D., in which the Hindu forms are given as a type of alphabet. The work is by one Ibn Wahshiyya, who is notorious as being one of the earliest nature fakirs, having written a monumental treatise purporting to explain Babylonian agriculture and civilization. This has been shown to be the product of an overactive imagination. While the treatise on alphabets is similarly largely fictitious, yet he does therein present the numeral forms now used by the Arabs.

32. In a continuous variety of $m$ dimensions are defined $m$ one-valued functions $x_{i}$ whose jacobian vanishes only on certain singular surfaces of not more than $m-1$ dimensions. The surfaces representing constant values of these functions divide the variety into regions bounded by $x_{i}=c_{i}, x_{i}=$ $c_{i}+\Delta c_{i}$. In one of these regions take $\Delta x_{i}=\Delta c_{i}$. By a continuous motion (birational) it will be possible to transform this into any other region except one bordering on the singular surface or boundary, in such a way that $x_{i}=c_{i}$ passes into $x_{i}=c_{i}{ }^{\prime}$. The increment of $x_{i}$ for this region is that into which $\Delta x$, passes by continuity. If the variety is bilateral the resulting quantity $\Delta x_{1} \cdots \Delta x_{n}$ will be independent of the path by which we pass (within the variety) from the first to the second point. In this case Dr. Phillips defines the integral of a one-valued function over the variety as

$$
\lim _{\Delta x=0} \Sigma f \Delta x_{1} \cdots \Delta x_{n}
$$


if that limit exists. If the variety is unilateral there are two values for the product of increments, the two differing only in sign. In this case both are used in the above limit and $f$ allowed to be correspondingly two-valued. With this definition of integration the formulas for change of variable are not limited to regions in which the jacobian has a constant sign. Symbolically the differentials under the integral sign multiply according to the alternative law. A series of results analogous to the formulas of Stokes and Gauss are given by the formula

$$
\int \cdots \int f d x_{1} \cdots d x_{n}=\int \cdots \int d f d x_{1} \cdots d x_{n},
$$

the one integral being taken over the boundary and the other over the region enclosed.

33. In this paper, Dr. Lipke characterizes geometrically the complete family of $\left(\infty^{2}\right)$ isogonal trajectories of a simple $\left(\infty^{1}\right)$ system of curves on any surface. (This has already been done for the plane by Professor Kasner and Dr. W. M. Smith.) To test whether a given family $(X)$ of $\propto^{2}$ curves on a surface are the isogonal trajectories of some simple system, we proceed as follows: Through each point in each direction there pass one curve of the family $(X)$ and one curve of the family $(I)$ of isogonal trajectories of an arbitrary isothermal system of curves; through each point in each direction construct a curvature element whose geodesic curvature is equal to the difference of the geodesic curvatures of the elements of $(X)$ and $(I)$ passing out in that direction, and rotate each new element through a right angle; if the family of curves thus obtained have the properties of a natural family of curves, then the family $(X)$ are the isogonal trajectories of a simple system. Natural families of curves on a surface have been geometrically characterized by the writer in a previous paper.*

34. This paper by Professor Shaw is a generalization of the integral invariants of Poincaré to expressions of a general vector analysis. The expressions are analogous to the quaternion generalizations of Green's theorems and similar forms. See Goursat, Journal des Mathématiques, (6) 4 (1908), page 331 .

* "Natural families of curves in a general curved space of $n$ dimensions." Trans. Amer. Math. Soc., vol. 13, p. 93. 
35. By non-linear algebra is meant one in which the coordinates of the product of two general numbers of the algebra are not bilinear forms in the coördinates of the two numbers, but are forms of higher order. Professor Shaw discusses the application of continuous transformation groups to a class of non-linear algebras.

36. In a paper read at the April, 1912, meeting of the Chicago Section, Professor Curtiss indicated the existence of other functions besides $e^{z x}$ which have the property, proved by Laguerre for the function named, provided $z$ is sufficiently large, that when the product of one of them with a polynomial $f(x)$ is developed in a power series, the number of variations of sign in the coefficients is equal to the number of positive real roots of $f(x)$. Since that time a paper has been published by Fekete and Pólya, proving that $(1-x)^{-k}$ is a function of this class for the interval $[0,1]$, provided $k$ is sufficiently large, and stating that $(1+x)^{k}$ has the same property for the interval $[0, \infty]$. Their demonstrations depend on systems of somewhat complicated inequalities. In the present paper briefer proofs of a different nature are given, and certain corollaries are deduced, of which the following may serve as an example: If $k$ is sufficiently large the number of variations of sign in the sequence

$$
\frac{d^{k}}{d x^{k}}\left(\frac{f(x)}{x}\right), \quad \frac{d^{k}}{d x^{k}}\left(\frac{f(x)}{x^{2}}\right), \cdots,
$$

for $x=x_{1}>0$, is equal to the number of positive roots of $f(x)$ less than $x_{1}$.

37. Professor Ranum considers the classification of $n$ dimensional spreads from a broad projective differential standpoint. For instance, ruled non-developable surfaces, which in $S_{3}$ may be regarded as all of the same class, in $S_{4}$ fall into four distinct classes; and non-developable hypersurfaces generated by $\infty^{1}$ planes in $S_{4}$ belong to four classes, while in $S_{5}$ they belong to forty-three classes. Analytic criteria are found for distinguishing the various classes.

38. Every complete set of conjugate substitutions, or subgroups of a simple group, is transformed according to a 
simply isomorphic group, whenever these substitutions or subgroups differ from identity. This characteristic property of simple groups may be used to define such groups.

In this paper Miss Schottenfels assumes the existence of the alternating group of degree 7 , and proves that if another simple group of this order exists, it contains the same number of Sylow subgroups of orders 7 and 9 as this alternating group. She then shows that the Sylow subgroups of order 9 in the supposed simple group are transformed according to an imprimitive group, and that these systems of imprimitivity are transformed according to the alternating group of degree 7. It follows that the supposed simple group is simply isomorphic with the alternating group of degree 7 , and that there exists but one simple group of order $7 ! / 2=2,520$.

Since it is known that there exist but one simple group of the orders 60 and 360, this paper establishes the fact that the alternating group of degree 8 is the smallest alternating group whose order is that of a non-alternating simple group (as the writer proved several years ago).

39. Professor Eisenhart's paper is essentially the same as that read at the Fifth International Congress, an abstract of which appeared in the January number of the Bulletin (page 177). The memoir will be printed in the Transactions.

40. The first explicit set of postulates for the notion "between" was given by Pasch in 1882, but no attempt was made at that time to establish the independence of the postulates. Substantially the same set was adopted by Peano in 1894, but again with no satisfactory proof of independence. Two later sets of postulates have been given, one by Hilbert in 1899, and one by Veblen in 1904; but neither of these sets is sufficient to establish some of the simplest laws of order along a straight line without the use of a certain two-dimensional existence postulate (the "triangle transverse postulate"), which was taken from the geometry of the plane and has properly speaking no place in a one-dimensional theory. The object of Professor Huntington's paper is (1) to supply complete proofs of independence for a set of postulates substantially the same as the original set of Pasch, and (2) to show how all the fundamental formal laws of serial order can be deduced from these postulates without the aid of any existence postulates. 
The postulates are as follows, where " $X \mathrm{R} A B$ " may be read " $X$ between $A$ and $B$." (1) If $X \mathrm{R} A B$, then $X \mathrm{R} B A$. (2) If $X \mathrm{R} A B$, then $A \neq B$. (3) If $X \mathrm{R} A B$ is true, then $A \mathrm{R} B X$ is false. (4) If $A \mathrm{R} X B$ and $B \mathrm{R} A Y$, and $X \neq Y$, then $A \mathrm{R} X Y$. (5) If $X \mathrm{R} A B$ and $B \mathrm{R} A Y$, and $X \neq Y$, then $X \operatorname{R} A Y$. (6) If $X \operatorname{R} A B$ and $Y \operatorname{R} A B$, and $X \neq Y$, then either $X \mathrm{R} A Y$ or $Y \mathrm{R} A X$. (7) If $A \mathrm{R} X B$ and $A \mathrm{R} Y B$, and $X \neq Y$, then either $X \mathrm{R} Y A$ or $Y \mathrm{R} X A$.

41. The present paper proposes to apply to the problem of developing an infinite determinant a procedure for postulating well ordered types which Professor Frizell communicated to the Fifth International Congress under the title: "Axioms of ordinal magnitudes." This consists essentially of a recurring process whereby, having built up a certain ordinal type $v=f(\tau)$, where $\tau$ is a previously defined ordinal magnitude, it is possible to postulate the new type $v=f(\tau+1)$ by using $v=f(\tau)$ as a basis after the following manner: The first value of $v$ is $f(1)=\omega$ and the postulating process assigns to it a well ordered set of symbols comprehending a set equivalent to the assemblage of all finite products of elements taken from the infinite matrix in accordance with the rule for forming a term of the determinant.

The result reached is to justify an analogous statement for subsequent values of $v$; and, since the factors of each product always form a series of type not lower than $\tau$, it follows that by carrying on the process up to $\tau=\omega_{1}$ we obtain terms of the infinite determinant arranged in a series the ordinal type of which is not higher than $f\left(\omega_{1}\right)$.

42. Let the analytic function $f\left(x_{1}, x_{2}, \cdots, x_{n}\right)$ be regular in a space $T$ of $2 n$ dimensions, and $a_{1}, a_{2}, \cdots, a_{n}$ be a point in this space where $f=0$. Then Weierstrass's preparation theorem gives the decomposition, in the vicinity of $a_{1}, a_{2}, \cdots, a_{n}$, of $f\left(x_{1}, x_{2}, \cdots, x_{n}\right)$ in two factors, one of which is a polynomial in $x_{1}-a_{1}$ and defines all the zeros of $f$ in the vicinity considered, except when the point $a_{1}, a_{2}, \cdots, a_{n}$ is such that $f\left(x_{1}, a_{2}, \cdots, a_{n}\right)$ vanishes identically in respect to $x_{1}$. In the case of exception it is necessary, according to Weierstrass, to perform a linear substitution on $x_{1}, x_{2}, \cdots, x_{n}$, the coefficients of which depend on $a_{1}, a_{2}, \cdots, a_{n}$.

Dr. Gronwall shows that it is possible to find, a priori, a 
linear substitution $x_{i}=\sum_{k=1}^{n} \alpha_{i k} x_{k}{ }^{\prime}(i=1,2, \cdots, n)$ such that, making $f\left(x_{1}, x_{2}, \cdots, x_{n}\right)=F\left(x_{1}{ }^{\prime}, x_{2}{ }^{\prime}, \cdots, x_{n}{ }^{\prime}\right)$, the expression $F\left(x_{1}{ }^{\prime}, a_{2}{ }^{\prime}, \cdots, a_{n}{ }^{\prime}\right)$ will not vanish identically in respect to $x_{1}{ }^{\prime}$ at any point $a_{1}{ }^{\prime}, a_{2}{ }^{\prime}, \cdots, a_{n}{ }^{\prime}$ in the space $T^{\prime}$ corresponding to $T$ by the substitution referred to, so that the case of exception will never occur for the new variables.

It is further shown how this theorem may serve to abbreviate materially a considerable number of proofs in the theory of analytic functions of several variables.

43. Dr. Gronwall has shown (in a paper presented to the Society, September, 1912) that for any absolutely integrable function $f(\theta, \varphi)$ the formal development in a Laplace series of spherical harmonics is summable by Cesàro's means of order one, with the sum $f(\theta, \varphi)$, in every point where the function is continuous. The present paper gives an investigation of the corresponding Cesàro means of order $k(0<k<1)$, and it is shown that for $\frac{1}{2}<k<1$ these means converge towards $f(\theta, \varphi)$ in any point $\theta, \varphi$ where the function is continuous, provided a certain limit expression vanishes at the antipode $\pi-\theta, \varphi+\pi$ (which is the case, for instance, when the function is bounded in the vicinity of this point). It is shown by examples that this restriction relative to $\pi-\theta, \varphi+\pi$ is also necessary. For $0<k \leqq \frac{1}{2}$, there exist functions $f(\theta, \varphi)$, continuous on the entire sphere, for which the Cesàro means of order $k$ are divergent at a given point.

Concerning the particular case of the Legendre development of a function $f(x)$ (where $x=\cos \theta$ ), continuous for $-1 \leqq x$ $\leqq 1$, it is shown that the corresponding Cesàro means of order $k$ (where $0<k<1$ ) converge towards $f(x)$ for -1 $<x<1$, but not necessarily at the end points $-1,+1$ of the interval.

44. The first part of the paper by Dr. Hennel deals with the general linear homogeneous difference equation of order $n$. The most general point transformation that changes every equation of this type and order into another of the same type and order is determined, and fundamental sets of seminvariants, invariants, semi-covariants, and covariants of the equation with regard to the group of point transformations are found. The second part of the paper is a similar discussion of a certain general type of functional equations. 
45. In the theory of numbers with Gauss the problems have to do with integers which are usually considered with regard to one number, an integer or modulus; so that the fundamental concept is a comparison of two numbers. These numbers may be represented by the coördinates of points in the plane, one of the coördinates being a fixed integer. A generalization of this idea, whereby the present scope of natural numbers is essentially widened, is a comparison of three or more integers in such a way that the relations among several integers may be expressed by the coördinates of points in a three or more dimensional space.

In Professor Hancock's first paper the Eulerian $\varphi$-function is generalized, other functions are introduced by means of which many new theorems in the theory of numbers are derived and certain theorems of Kronecker, Dedekind, Lipschitz, Cesàro, and others are extended.

46. In Professor Hancock's second paper is generalized the theorem stated by Kronecker in his Vorlesungen über Zahlentheorie, page 250. Among the applications it is shown that

$$
\begin{array}{r}
\sum_{n=1}^{n=N} \sum_{k=1}^{k=K} \sum_{(n, \kappa, \tau) \sim 1} \tau=\frac{1}{6} \sum e_{d}\left[\frac{K}{d}\right] d d^{\prime}\left(d^{\prime}+1\right)\left(d^{\prime}+2\right) \\
\tau \leqq n \quad\left(d d^{\prime} \leqq N ; d=1,2, \cdots, N\right)
\end{array}
$$

where $e_{d}$ are the Möbius coefficients, $[K / d]$ represents the greatest integer in $K / d$, and $(n, \kappa, \tau)$ denotes the greatest common divisor of $n, \kappa$, and $\tau$.

47. About a year ago Professor Wilczynski announced the theorem that isosceles triangular solutions of the problem of three bodies, other than the Lagrangian equilateral triangular solutions, do not exist unless two of the masses are equal. Professor MacMillan shows that if a solution exists the expressions for the orbits in polar coördinates are readily obtained from the integrals of areas. The expressions thus obtained must satisfy a certain linear differential equation of a very simple type. The proof is obtained by showing that if the masses are all different the singularities of these expressions cannot be made to coincide with the singularities of this linear differential equation by any choice of the available 
constants except such as give the equilateral triangular solution.

48. In 1896 Poincaré pointed out a defect in the proof of Brun's theorem of the non-existence of algebraic integrals in the problem of three bodies. Poincaré pointed out also the method by which this defect could be remedied, but did not give the details of his analysis. The details, given by several other writers, are not entirely satisfactory and it is the purpose of Professor MacMillan's second paper to remedy these deficiencies.

49. The principal results in Professor Fite's paper are contained in the following theorems:

If $G$ is a group of class $k$ and order $p^{m}, p$ an odd prime, its second central cannot be cyclic.

If the commutator subgroup of $G$ is cyclic, its $(k-1)$ th central cannot be abelian, when $k>3$.

If the commutators of $G$ that correspond to the invariant operations of the first cogredient of $G$ form a cyclic subgroup, then the commutator subgroup of $G$ is cyclic.

This last theorem is closely related to a theorem of Professor Burnside's in a recent number of the Proceedings of the London Mathematical Society to the effect that a non-abelian group whose central is cyclic cannot be a commutator subgroup of a group of order $p^{m}$.

50. Various definitions have been given of the "sum" of a divergent series (cf. Cesàro, Riesz, Borel, LeRoy, etc.). In this paper, Mr. Smail gives a process which leads to four general methods of summation of divergent series, and each of these four methods includes as special cases several of the known definitions. It is shown that all these general methods satisfy the so-called "condition of consistency," i. e., that every convergent series is summable with generalized "sum" equal to the ordinary sum; that no properly divergent series is summable by these methods. Uniform summability, the continuity, and the term-by-term integration and differentiation of uniformly summable series are discussed. Applications are then made of these general theorems to the various particular known methods (Cesàro's, Riesz's, Borel's, LeRoy's, and the Cesàro-Riesz methods), resulting in many known 
theorems as well as many new theorems. The methods of proof employed throughout are simpler than those hitherto used for proving the theorems directly for the particular methods. In this way the essential properties of the various known methods are brought out, and also greater uniformity of treatment is secured.

51. It is known that if the coefficients of a homogeneous linear differential equation are developable in asymptotic power series for large (real) values of the independent variable, then a set of fundamental solutions of the equation likewise possess asymptotic expansions in the same region, provided the roots of the so-called auxiliary equation are distinct. The chief object of Mr. Love's paper is to study the case in which the roots of the auxiliary equation are not all distinct. The method used is an application of a general theorem arising from Dini's researches in the theory of linear differential equations. For the equation of arbitrary order, only an incomplete discussion is attempted, on account of the multiplicity of cases that arise, but the equation of the second order is considered in detail, and for this the problem is completely solved, leading to six distinguishable cases.

52. In this paper Professor Snyder gives two examples of surfaces of any order and of arbitrary geometric genus (or of index of irregularity) that have infinite groups of birational transformations that are Cremonian groups for entire space. The paper appeared in full in the January number of the Transactions.

53. In a paper previously read before the Society,* Dr. Silverman has given a generalization of all the definitions of summability of a certain type. In the present paper, sufficient conditions are obtained for the equivalence of any two such general definitions. Several new theorems are obtained concerning Cesàro's and Hölder's definitions; and the new proof given for the equivalence of Cesàro's summability of order $r$ and Hölder's summability of order $r$ seems simpler than the proofs previously given. $\dagger$

* October, 1910.

† Ford, American Journal, 1909; Schnee, Math. Annalen, 1909. 
54. The varieties of self-projective quartic and quintic curves have been tabulated for the general case by Ciani and Snyder respectively. Dr. Winger in his paper presents the projectively distinct types of the most general rational curves of these orders which are invariant under the different finite collineation groups. The quartics are readily obtained from the consideration of the Stahl binary sextic. Six types are found with characteristic groups of orders $2,3,4,4,6$ and 24 , the first three being cyclic, besides one with an infinite group.

Of the quintics, two admit a one-parameter group. The others belong to cyclic groups of orders $2,3,4,5$ (two types), and dihedral groups of orders 4, 6, and 10 (two types),--eleven in all.

F. N. Cole, Secretary.

\section{THE PRODUCT OF TWO OR MORE GROUPS.}

BY PROFESSOR G. A. MILLER.

(Read before the American Mathematical Society, December 31, 1912.)

\section{§1. Introduction.}

If $H_{1}$ and $H_{2}$ are any two groups, the symbol $H_{1} \cdot H_{2}$ denotes the totality of the products obtained by multiplying each operator of $H_{1}$ on the right by every operator of $H_{2}$. A necessary and sufficient condition that this totality constitutes a group is that $H_{1} \cdot H_{2} \equiv H_{2} \cdot H_{1}$. As $H_{1} \cdot H_{2}$ is always composed of the inverses of all the operators represented by $H_{2} \cdot H_{1}$, irrespective of whether this product is a group or does not have this property, we may also say that a necessary and sufficient condition that $H_{1} \cdot H_{2}$ is a group is that it includes the inverse of each one of its operators.

Suppose that $H_{1}$ and $H_{2}$ have exactly $h_{0}$ operators in common. These common operators constitute a subgroup $H_{0}$, which is known as the cross-cut of $H_{1}$ and $H_{2}$. It is easy to prove that the number of the distinct operators in $H_{1} \cdot H_{2}$ is always $h_{1} h_{2} / h_{0}$, where $h_{1}$ and $h_{2}$ represent the orders of $H_{1}$ and $H_{2}$ respectively. To see that $H_{1} \cdot H_{2}$ cannot involve more than this number of distinct operators, it is only neces- 